\title{
Design of Wireless Optical Access System using LED
}

\author{
Liwei Ding, Fang Liu, Yingjie He, Hongbo Zhu, Yongjin Wang \\ Institute of Communication Technology, Nanjing University of Posts and Telecommunications, Jiangsu, China \\ Email: wangyj@njupt.edu.cn
}

Received 2013

\begin{abstract}
This paper develops a prototype to demonstrate a full-duplex wireless visible light communication (VLC) system based on Universal Serial Bus (USB) port. Combing with a USB 2.0 port on one board, it can achieve up to 2 Mbps bit rates error free, which is mainly limited to the USB bridge circuit, between two computers for data transmission, such as text, audio and video. Hyper Terminal and self-written software are used to allow setting the transmission data rate, control bits, check bits and achieve real-time transmission between any two mobile devices with a USB port. The work is based on the research of VLC local access network. This paper also shows the experimental results and the relationship between system transmission eye diagram and bit rate.
\end{abstract}

Keywords: Visible Light Communications; Visible Light Access Networking; USB Interface; Real-time Transmission; Full-duplex

\section{Introduction}

Looking into the future, traditional home and office illumination tools will soon be substituted by the lower energy consuming, longer lifetime and health harmless LED devices. Meanwhile, the very fast changing capability in the output power makes LED devices possible to transmit a large amount of data at high speeds in the visible range, which is an added advantage of LED besides its illumination purpose. Visible light communication (VLC) using white LEDs has aroused global attention. At present, many famous research institutions and universities, such as the Visible Light Communications Consortium (VLCC) [1-2], the European OMEGA project [3], the Wireless World Research Forum (WWRF) [4] and so on, have dedicated much to area. And it has witnessed increasing interest in the research community with a forthcoming IEEE standard [5]. High speed transmission rate [6,8], channel characteristics [9,10], modulation and demodulation methods [11-13], etc [14,15] are the main focuses of research.

USB is a universal standard of external bus to specify the connection and communication through computer and peripheral electronic devices. USB port as the interface to PC with its popularity and support to hot plugging. VLC with a variety of practical applications in shortrange transmission scenarios is promised to enter into people's daily life. The channel between two computers via VLC has been shown in [16-19], where RS-232 protocol or RS-485 protocol is used to connect visible light module with a PC. Design of an integrated optical re- ceiver using USB interface is introduced in [20]. The data rate and transmission distance can surely be improved by increasing the number of LEDs and adopting modulation schemes. An application scenario for the developed system is indoor short distance wireless transmission where LED with communication function is enveloped as a desk lamp and when turned on, data stream flows wirelessly in both ways.

In this paper, a full-duplex access system using white LED based on USB port with 2 Mbps data rate is designed as a basic visible light access network. Hyper Terminal and self-written software are used to achieve the transmission of data and real-time audio transmission between two PCs. The rest of this paper is organized as follows. Section 2 gives the system design and system characteristics. In section 3, the testing and results of transmission link is discussed. Finally, Section 4 concludes the paper.

\section{System Design and System Characteristics}

\subsection{System Design}

A CH340 is used to connect USB interface for realizing the conversion of signal from differential to TTL, or inverse. USB signal after conversion has a $4 \mathrm{~V}$ peak-topeak voltage value. A voltage follower then plays the role of buffer to amplify the signal current to an adequate level, driving LED for lighting. A high power white LED of $1 \mathrm{~W}$ and with the beam angel $120^{\circ}$ is used as optical transmission source. OOK NRZ data for Intensity modulation (IM) and direct detection (DD) scheme is chosen 
for the widely reception in VLC research. The circuit design of transmitter is given as Figure 1.

The receiver employs a commercially available Si PIN (Hamamatsu S6968) photodiode for optical to electrical conversion with $150 \mathrm{~mm}^{2}$ effective active area, $14 \mathrm{~mm}$ active area size and $35^{\circ}$ half angle. The received optical signal is sent to a low pass filter followed by a custom amplifier for recovery. A decision circuit (LT1715) is used as a single threshold voltage comparator to achieve the signal decision with a reference voltage level to obtain the original signal. The TTL signal after decision is sent to CH340 to achieve the conversion from TTL signal to USB serial signal. The whole circuit of receiver design is given in Figure 2. It needs to be noted that the transceiver module is composed of a transmitter and a receiver to realize a full-duplex wireless communication. Transceiver design is shown in Figure 3.

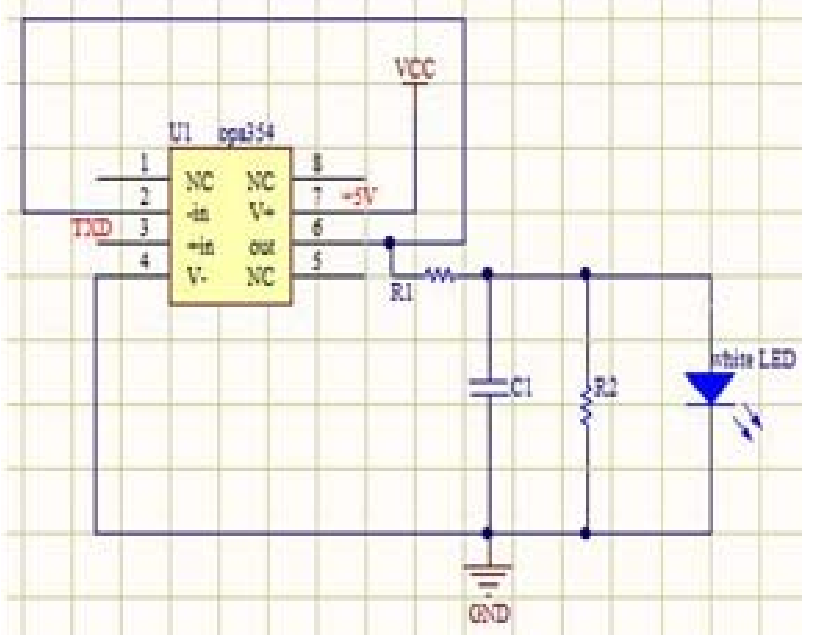

Figure 1. Transmitter circuit diagram.

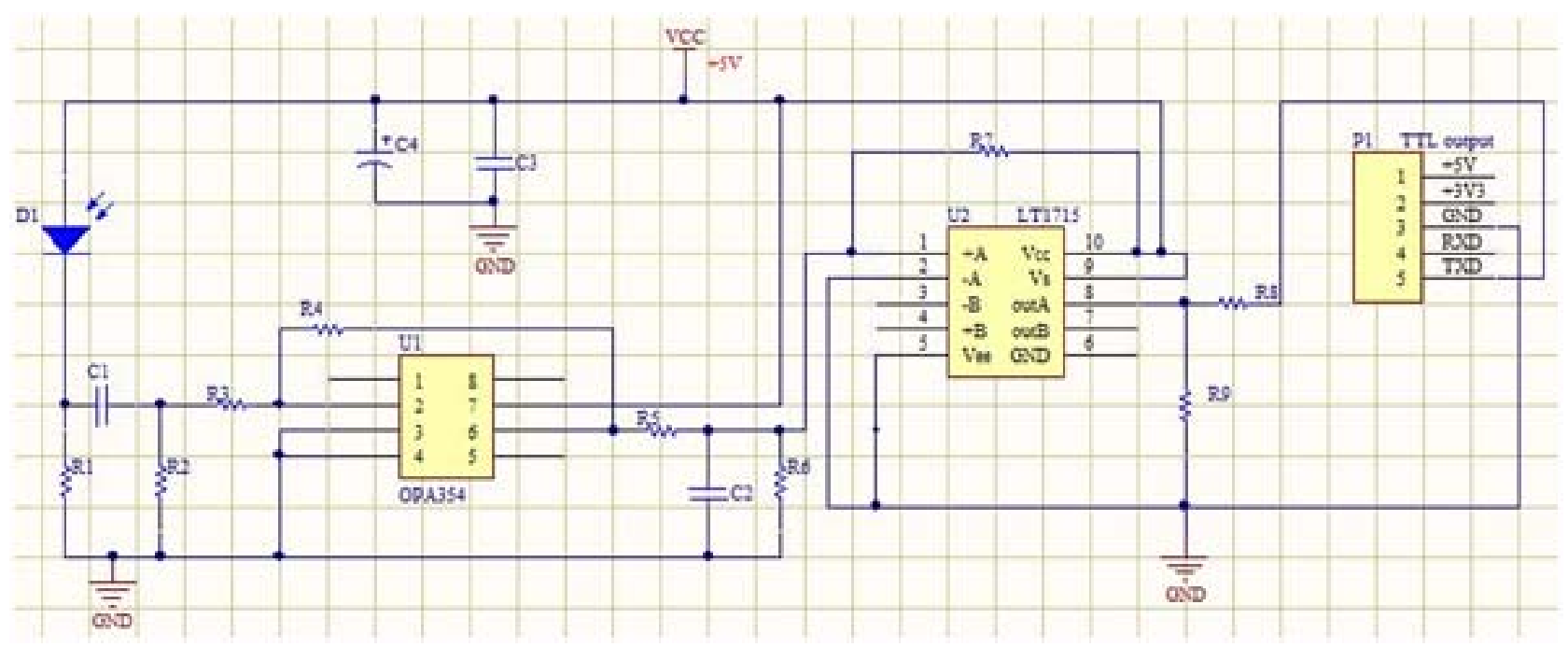

Figure 2. Receiver circuit diagram.
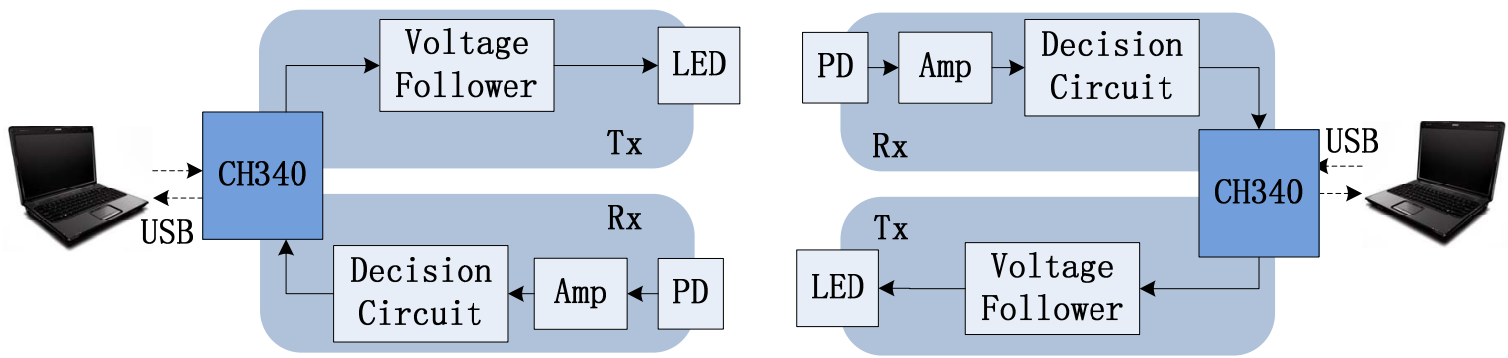

Figure 3. Transceiver block diagram.

\subsection{System Characteristic}

All the system power supply is supported by the 5V DC from USB port. The driver of CH340 must be firstly installed on PC before being connected to a transceiver module. After transmission rate being set and Com port being selected in Hyper Terminal, which is a communi- cation tool available in computer's self-carried enclosures, PC treats the transceiver module as a visual serial port via which all types of the data can be transmitted, such as text, audio, video and so on. Another application example is developed using Visual C\#. Program is created to achieve real time audio transmission between two transceiver-settled computers. The input voice from mi- 
crophone or music of wave waveform type is captured by one computer, and then audio data is sent to the selected Com port and transmitted by our transceiver module. After a light of sight channel, the opposite transceiver module interfaced on the second PC received the bit streams and played it real time. The physical map of the system model is shown in Figure 4. The transmission between two computers using Hyper Terminal is shown in Figure 5.

\section{Testing and Results}

With the VLC system described above, performance of the transceiver module is measured. On one hand, we studied the eye pattern of the TTL signal before decision circuit versus data rate, and the relationship between data rate and eye diagram is investigated. On the other hand, the text transmission using program based on Visual C\# is also given. The system is tested using the configuration shown in Figure 6, as the arbitrary waveform generator and oscilloscope instead of PCs.
Figure 7 presents the eye diagrams for the receiver arbitrary OOK NRZ data (PN23) at speeds of $1 \mathrm{Mbps}$ and 2 Mbps after optical signal conversation. The eye diagrams of the signal receive without amplify and filtering are clear, thus show error free performance. The eye diagrams indicate that the signal can be correct recovered after decision circuit.

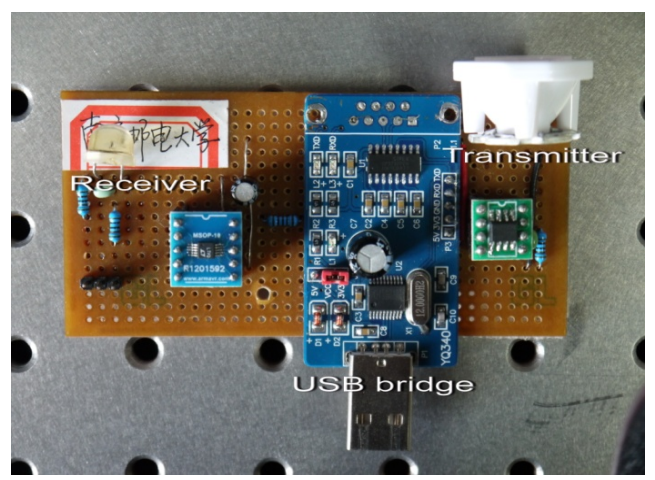

Figure 4. Physical map of system design.

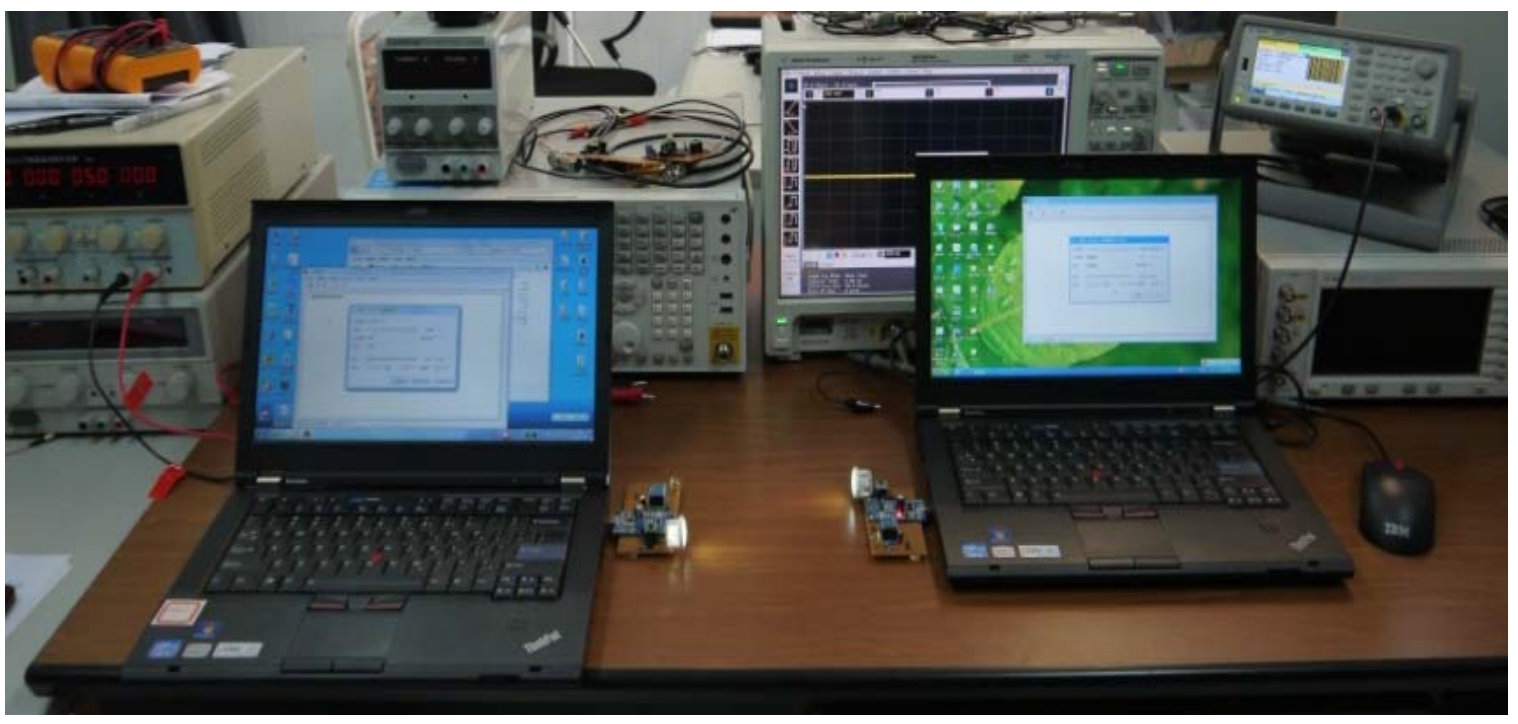

Figure 5. Prototype of the full-duplex system.

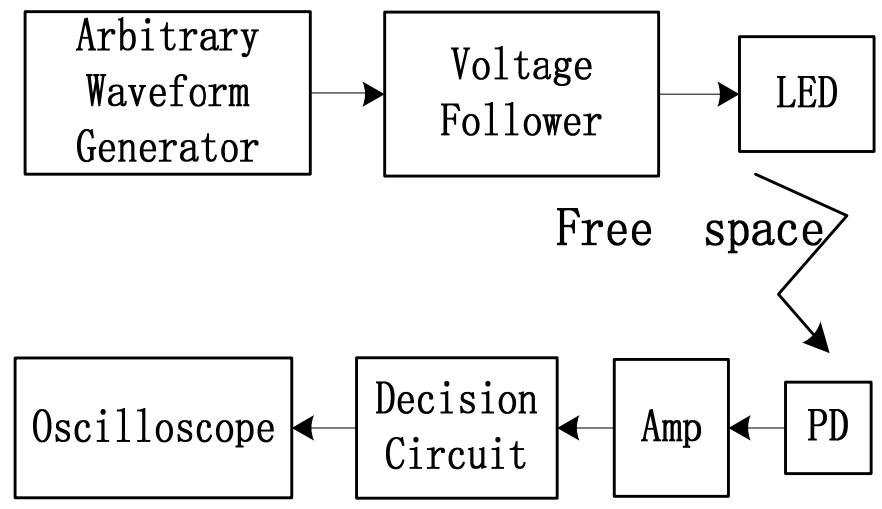

Figure 6. Testing block diagram. 

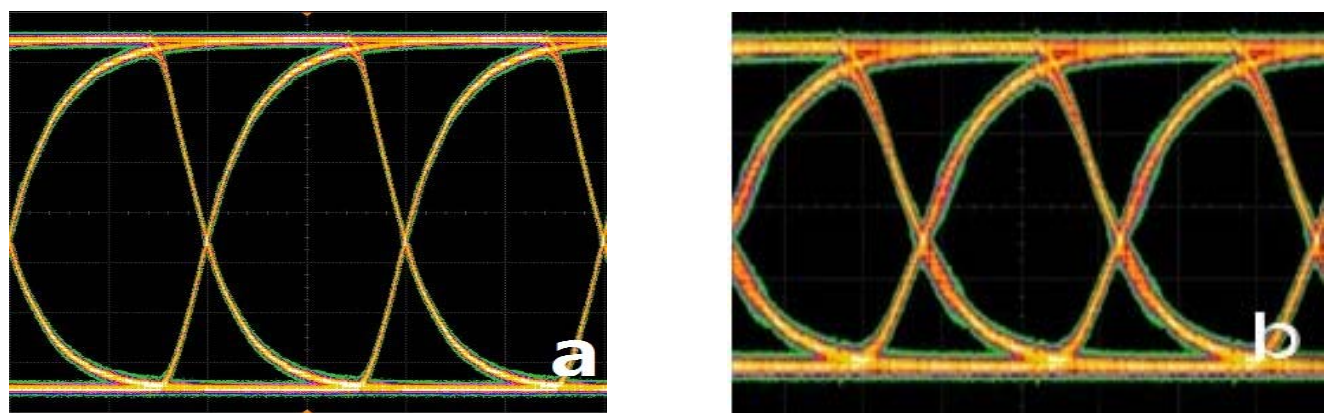

Figure 7. Eye diagram waveforms after direct detection without filtering and being amplified at (a) 1 Mbit/s data rate, (b) 2 Mbit/s data rate.
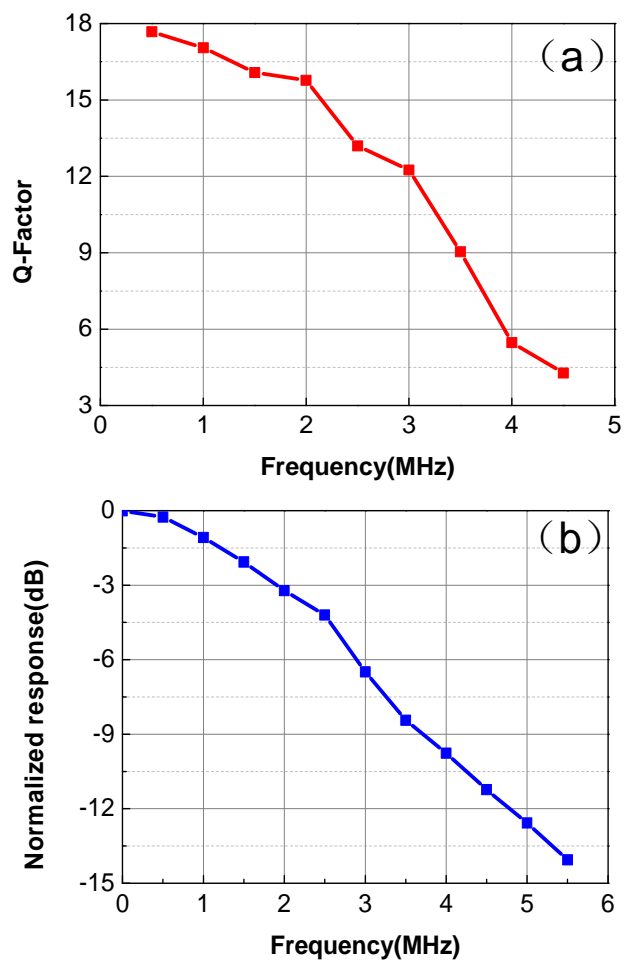

Figure 8. (a) Eye diagram Q-factor Vs data rate, (b) frequency response curves of the whit light LED.

We obtained the Q-factor of eye diagram at different transmission rates and estimated the BER from Q-factor. All BERs are below $10^{-6}$ when data rate goes up to 4.5 Mbit/s. The curve of Q-factor vs data rate is showed in Figure 8(a). We can find that the BER performance is mainly restricted to $3 \mathrm{~dB}$ bandwidth of the white LED emitter, where the normalized response is shown in Figure 8(b).

In this experiment, the system of VLC was demonstrated for applying to data transmission because the concept of VLC can be used in marine, automotive, underwater, home network and other scenarios. The green waveforms stand for the transmission signal and the yellow waveforms come from the receiver after decision circuit, as Figure 9 shown. It is clear that no bit error
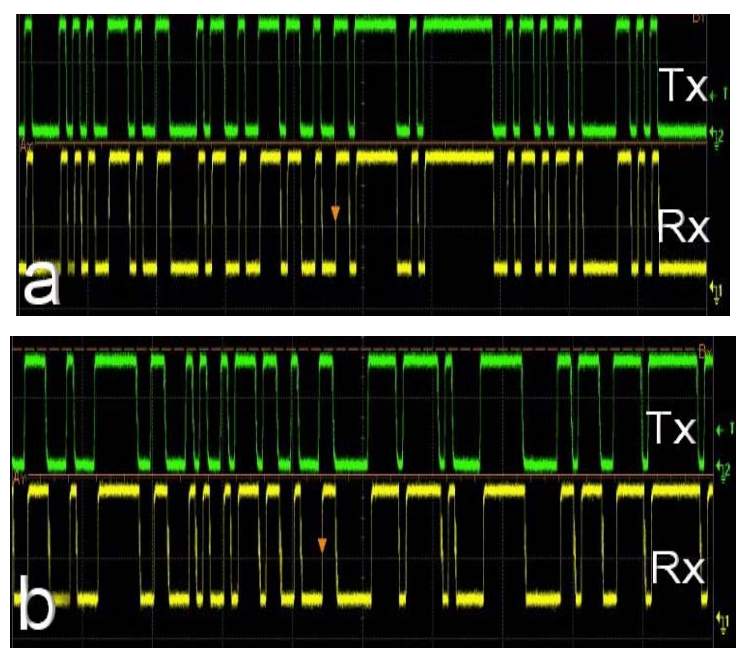

Figure 9. Captured data waveforms after decision circuit at (a) $1 \mathrm{Mbit} / \mathrm{s}$ data rate, (b) $2 \mathrm{Mbit} / \mathrm{s}$ data rate.

occurs after visible light transmission through the system and the decision circuit can correctly complete the signal decision.

\section{Conclusions and Future Work}

We developed a full-duplex optical wireless system prototype to achieve the transmission between two computers using white light LED. The upper limit transmission rate is $2 \mathrm{Mbit} / \mathrm{s}$, which is restricted to USB bridge circuit CH340. The obtained Q-factor of eye diagram at different transmission rates and estimated the BER from Q-factor. All BERs are below 10-6 when data rate goes up to $4.5 \mathrm{Mbit} / \mathrm{s}$. The higher USB bridge circuit, the more optimizing circuit designs, efficient coding method, advanced modulation techniques and mechanism for correcting errors will be considered to improvement the transmission system in our future work.

\section{Acknowledgements}

This work is jointly supported by NSFC (11104147), Jiangsu 973 project (BK2011027), and research project (NY211001, BJ211026). 


\section{REFERENCES}

[1] VLCC, "Visible Light Communications Consortium," 2008.

[2] M. Nakagawa, "Visible Light Communications," 2007.

[3] "Home Gigabit Access project," funded by European Framework 7." http://www.ict-omega.eu/.

[4] "Wireless World Research Forum." http://www.wireless-world-research.org/.

[5] IEEE, "IEEE P802.15 Working Group for Wireless Personal Area Networks (WPANs),” 2008.

[6] J. Vucic, C. Kottke, S. Nerreter, K. Habel, A. Buttner, K. D. Langer and J. W. Waleski, "125 Mbit/s over $5 \mathrm{~m}$ Wireless Distance by Use of OOK-Modulated Phosphorescent White LEDs," Processing of 35th European Conference of Optical Communication, 20-24 September 2009.

[7] H. Le-Minh, D. O’Brien, G. Faulkner, L. Zeng, K. Lee, D. Jung, Y. Oh and E. T. Won, "100-Mb/s NRZ Visible Light Communications Using a Postequalized White LED,” IEEE Photonics Technology Letters, Vol. 21, No. 15, 2009.

[8] Y. Zheng and M. Zhang, "Visible Light Communications Recent Progresses and Future Outlooks," Processing of Photonics and Optoelectronics Conference, 2011, pp. 1-6.

[9] T. Komine and M. Nakagawa, "Fundamental Analysis for Visible-Light Communication System using LED Lights," IEEE Transactions on Consumer Electronics, Vol. 50, No. 1, 2004, pp. 100-107. doi:10.1109/TCE.2004.1277847

[10] L. Zeng, D. O’Brien, H. Le-Minh, G. E. Faulkner, K. Lee, D. Jung, Y. Oh, and E. T. Won, "High Data Rate Multiple Input and Multiple Output (MIMO) Optical Wireless Communications using White LED lighting,” IEEE Journal Selected Areas in Communications, Vol.27, No.9, 2009, pp.1654-1662. doi:10.1109/JSAC.2009.091215

[11] R. Mesleh, H. Elgala and H. Hass, "Optical Spatial Modulation," Journal of Optical Communications and Networking, Vol. 3, No. 3, 2011, pp. 234-244. doi:10.1364/JOCN.3.000234
[12] Z. Wang, C. Yu, W. D. Zhong and J. Chen, "Performance Improvement by Tilting Receiver Plane in M-QAM OFDM Visible Light Communications,” Optics Express, Vol. 19, No. 14, 2011, pp. 13418-13427. doi:10.1364/OE.19.013418

[13] C. H. Yeh, Y. F. Liu, C. W. Chow, Y. Liu, P. Y. Huang and H. K. Tsang, "Investigation of 4-ASK Modulation with Digital Filtering to Increase 20 Times of Direct Modulation Speed of White-Light LED Visible Light Communication System,” Optics Express, Vol. 20, No. 15, 2012, pp. 16218-16223. doi:10.1364/OE.20.016218

[14] B. Jin, M. Zhang, Y. Zhang and N. Hao, "Visible Light Communications using Blind Equalization,” Processing of Communications and Photonics Conference, 13-16 November 2011, Vol. 8309, 2011, pp. 83092N-1-83092N-6.

[15] G. Cossu, A. M. Khalid, P. Choudhury, R. Corsini and E. Ciaramella, "3.4 Gbit/s Visible Optical Wireless Transmission Based on RGB LED,” Optics Express, Vol. 20, No. 26, 2012, pp. B501-B506. doi:10.1364/OE.20.00B501

[16] K. Cui, G. Chen, Z. Xu and R. D. Roberts, "Ling-of-sight Visible Light Communication System Design and Demonstration,” CSNDSP, 2010, pp. 621-625.

[17] N. Shrestha, M. Sohail and C. Viphavakit, "Demonstration of Visible Light Communications Using RGB LEDs in an Indoor Environment," Processing of ECTI-CON International Conference, 19-21 May 2010, pp. 1159-1163.

[18] T. D. C. Little, P. Dib, K. Shah, N. Barraford and B. Gallagher, "Using LED Lighting for Ubiquitous Indoor Wireless Networking," IEEE International Conference on Wireless and Mobile Computing, 12-14 October 2008, pp. 373-378.

[19] Y. Yang, X. Chen, L. Zhu, B. Liu and H. Chen, "Design of Indoor Wireless Communication System Using LEDs," Communications and Photonices Conference, 2-6 November 2009, Vol. 7632, pp. 1-8.

[20] A. Burton, C. Amiot, H. L. Minh and Z. Ghassemlooy, "Design of an integrated Optical Receiver for Mobile Visible Light Communications,” PGNet, 2011. 